\title{
Differential Expression of Immunophilins FKBP51 and FKBP52 in the Frontal Cortex of HIV-Infected Patients with Major Depressive Disorder
}

\author{
Erick T. Tatro • Ian P. Everall • Eliezer Masliah • \\ Britta J. Hult • Ginger Lucero • Gursharan Chana • \\ Virawudh Soontornniyomkij • Cristian L. Achim • \\ HNRC
}

Received: 20 October 2008 / Accepted: 16 January 2009/Published online: 6 February 2009

(C) The Author(s) 2009. This article is published with open access at Springerlink.com

\begin{abstract}
Patients infected with human immunodeficiency virus (HIV) have a higher risk of developing major depressive disorder (MDD) than the general population. Immunophilins FKBP51 and FKBP52 are expressed in cortical neurons and regulate the function of the glucocorticoid receptor (GR). Previous reports have shown that genetic variants in the FKBP5 gene encoding FKBP51 are linked to psychiatric disorders. We sought to determine whether immunophilins are upregulated in HIV infection. To determine whether FKBP52 and FKBP51 are associated with MDD and/or HIV, we compared protein and gene expression in autopsy tissues from the frontal cortical gray matter. The study cases were divided into five groups: control, MDD, MDD with psychosis, $\mathrm{HIV}^{+}$, and $\mathrm{HIV}^{+}$with MDD. Gene expression and protein levels were determined by real-time PCR and Western blot analysis of fresh frozen tissues. Genotyping of previously published alleles of the FKBP5 gene was also performed. We found correlation of upregulation of both immunophilins in the HIV-infected groups. In the $\mathrm{HIV}^{+}$ population with MDD, FKBP4 expression is significantly
\end{abstract}

\author{
E. T. Tatro • I. P. Everall • B. J. Hult • G. Lucero • G. Chana • \\ V. Soontornniyomkij · C. L. Achim $(\square)$ \\ Department of Psychiatry-0603, \\ University of California at San Diego, \\ BSB\#2066, 9500 Gilman Drive, La Jolla, \\ San Diego, CA 92093-0603, USA \\ e-mail: cachim@ucsd.edu \\ E. Masliah \\ Department of Neurosciences, \\ University of California at San Diego, \\ San Diego, CA, USA \\ E. Masliah • C. L. Achim \\ Department of Pathology, University of California at San Diego, \\ San Diego, CA, USA
}

higher while $F K B P 5$ is more variable. After analyzing the $F K B P 5$ gene for single nucleotide polymorphisms, we found that rs3800373 CC genotype is more frequent in the MDD and MDD/Psychosis groups. We hypothesized that the levels of FKBP51, as modulator of the nuclear translocation of GR, would be lower in MDD. Instead, an increase in FKBP51 at both the transcript (FKBP5) and protein level correlated with MDD. Increased FKBP4 expression of correlated to $\mathrm{HIV}^{+} \mathrm{MDD}$ but not to HIV without MDD.

Keywords HIV - immunophilins ·

major depressive disorder $\cdot$ glucocorticoid receptor

\section{Introduction}

Immunophilins are a class of chaperone and adapter proteins widely expressed in most tissues, particularly abundant in the brain, specifically in neurons (Steiner et al. 1992; Sinars et al. 2003). FKBP52 and FKBP51 are the products of the FKBP4 and $F K B P 5$ genes, respectively. FKBP52 functions as an adapter protein within the glucocorticoid receptor (GR) and HSP90 complex that links the hormone-bound receptor complex to the molecular motor dynein allowing for shuttling of the activated receptor complex to the nucleus (Wochnik et al. 2005). In contrast to FKBP52, the FK506binding domain of FKBP51 lacks isomerase function, but is shown to bind the GR through the tetratricopeptide repeat domains and therefore functions as a competitive inhibitor to formation of the GR-FKBP52-dynein complex (Denny et al. 2000; Westberry et al. 2006). Sapolsky's glucocorticoidcascade hypothesis proposes a feed-forward mechanism of decreased feedback inhibition with increased sensitivity of the brain to cortisol (Sapolsky and Plotsky 1990). The action 
of the GR in the brain may be central to the molecular mechanism whereby the hypothalamo-pituitary-adrenal axis feedback loop is dysregulated in hypercortisolemia observed in depression (Sapolsky and Plotsky 1990; Garcia et al. 2004). Therefore, the modulation of GR signaling in the cortex of the brain may be a level of regulation that could affect the susceptibility of neurons to lose synaptic and dendritic density found in hypercortisolemia and depression (Cotter et al. 2002a, b).

Single nucleotide polymorphisms (SNPs) in the FKBP5 gene have been associated with MDD, posttraumatic stress disorder, treatment response to drugs, and delayed recovery from psychosocial stress in healthy adults (Binder et al. 2004, 2008; Ising et al. 2008; Lekman et al. 2008). The specific molecular consequences of these SNPs are not known. Binder et al. identified two polymorphisms that link to decreased FKBP51 protein levels in the blood (Binder et al. 2004). The same polymorphisms in FKBP5 were linked with peritraumatic dissociation in medically injured children (Koenen et al. 2005). However, one prospective study failed to definitively assign the designated SNPs' associations with MDD and affective disorder (Gawlik et al. 2006). FKBP5 may play a role only in some depression cases, with other genes involved in the glucocorticoid system responsible for disturbances of the HPA axis during depressive episodes.

Since the prevalence of major depressive disorder (MDD) is significantly elevated in individuals infected with HIV, we sought to determine whether there was a relationship between HIV infection, MDD, and the expression of immunophilins in the frontal cortex (Reiter 2000; Valente 2003; Porche and Willis 2006). We have previously showed an increase of the related immunophilin FKBP12 in the deep gray matter of HIV patients, which we hypothesized to be a protective response (Christeff et al. 1997; Bing et al. 2001; Valente 2003; Porche and Willis 2006).

\section{Materials and methods}

\section{Demographic information}

Autopsy fresh frozen brain tissues were kindly provided by the Stanley Foundation and the California NeuroAIDS Tissue Network (CNTN). Twelve cases from each group were studied with an age range of 24-63 years. The cases were divided into five study groups: control, MDD, MDD with psychosis, $\mathrm{HIV}^{+}$, and $\mathrm{HIV}^{+}$with MDD (Table 1). The cause of death in the control group was mainly cardiac arrest, while in the depressed groups, the cause of death was predominantly suicide. The cause of death for the two HIV groups was AIDS-associated complications. The postmortem interval (PMI) was generally within $24 \mathrm{~h}$ (the average in the CNTN cohort was approximately 12 and
$35 \mathrm{~h}$ for the Stanley Foundation cohort). One subject in the HIV/MDD group was diagnosed with HIV-associated dementia (HAD), no. 49, and two subjects in the HIV group were diagnosed with minor cognitive and motor deficit (MCMD), subjects no. 41 and no. 45.

\section{FKBP4 and FKBP5 gene expression}

For RNA isolation, $100 \mathrm{mg}$ was homogenized in ice-cold TRIzol reagent (Invitrogen) following manufacturer's instructions. RNA quality was assessed by the A260/A280 ratio and by agarose electrophoresis. CDNA synthesis was performed using $1 \mu \mathrm{g}$ of RNA using the SuperScript III kit from Invitrogen. For quantitative PCR, 40 ng cDNA per reaction was used, and FAM-labeled $20 \times$ prevalidated probes were purchased from Applied Biosystems. For FKBP5, assay Hs00188025_m1; for FKBP4, assay Hs00427038_g1; and for GAPDH, assay Hs02758991_g was used. TaqMan master mix $(2 \times)$ purchased from Applied Biosystems was used in $20 \mu \mathrm{L}$ reactions on 96 well plates, and assays were performed at the University of California San Diego Center for AIDS Research Genomics Core. Gene expression is reported as fold control versus the median of the control group using the $\Delta \Delta$-CT method comparing to housekeeping gene $G A P D H$ whereby $\Delta \mathrm{CT}=\mathrm{CT}_{\mathrm{GAPDH}}-\mathrm{CT}_{\mathrm{Gene}}, \Delta \Delta \mathrm{CT}=$ $\Delta \mathrm{CT}_{\text {Control }}-\Delta \mathrm{CT}_{\text {Disease, }}$ and Fold - Control $=2^{-\Delta \Delta \mathrm{CT}}$. A test for normality showed that not all groups are distributed in a Normal curve; therefore, nonparametric statistical analyses were performed, and Kruskal-Wallace rank sums test and Dunn's multiple comparison were done using Graphpad Prism software to compare the groups.

\section{FKBP52 and FKBP51 protein analysis}

For protein extraction, $100 \mathrm{mg}$ of frozen brain tissue was cut and homogenized in $0.9 \mathrm{~mL}$ ice-cold lysis buffer consisting of $50 \mathrm{mM}$ Tris- $\mathrm{HCl}, 1 \%$ sodium dodecyl sulfate, $150 \mathrm{mM} \mathrm{NaCl}$, $1 \mathrm{mM}$ EDTA, $1 \mathrm{mM}$ phenylmethylsulfonyl fluoride, $1 \mu \mathrm{g} / \mathrm{mL}$ aprotinin, $1 \mathrm{mM}$ sodium $\mathrm{Na}_{3} \mathrm{VO}_{4}$, $\mathrm{pH}$ 7.4. Five micrograms of total protein from each case was run in $10 \%$ polyacrylamide gels and transferred to polyvinylfluoridine membranes. Western blotting analysis for immunophilins FKBP52 and FKBP51 was performed using primary antibodies incubated overnight at $4^{\circ} \mathrm{C}$ : mouse anti-FKBP52 (Stressgen SRA1400) at 1:5,000 and rabbit anti-FKBP51 (Abcam ab2901) at 1:1,000. Secondary antibodies were horseradish peroxidaseconjugated donkey anti-mouse and donkey anti-rabbit (Jackson Immunoresearch), respectively, at 1:5,000. The chemiluminescence signal was developed according to manufacturer's protocols (Perkin Elmer NEL103001) and quantified using densitometric analysis with Image $\mathbf{J}$ software; changes are expressed as fold actin and normalized fold actin. 
Table 1 Demographic description of study groups

\begin{tabular}{|c|c|c|c|c|c|c|c|c|c|c|}
\hline Patient ID & Age & Sex & PMI & $\mathrm{pH}$ & $\begin{array}{l}\text { Cause of } \\
\text { death }\end{array}$ & Patient ID & Age & Sex & PMI & $\begin{array}{l}\text { Cause of } \\
\text { death }\end{array}$ \\
\hline Control & & & & & & $\mathrm{HIV}^{+} \mathrm{MDD}$ & & & & \\
\hline 1 & 48 & M & 12 & 6.51 & Cardiac & 49 & 43 & $\mathrm{M}$ & 12 & AIDS \\
\hline 2 & 24 & M & 17 & 6.6 & MVA & 50 & 43 & M & 31 & AIDS \\
\hline 3 & 50 & $\mathrm{~F}$ & 35 & 6.31 & Cardiac & 51 & 34 & $\mathrm{M}$ & 10 & AIDS \\
\hline 4 & 44 & $\mathrm{M}$ & 27 & 6.82 & AAP & 52 & 55 & M & 120 & AIDS \\
\hline 5 & 35 & M & 31 & 6.59 & MVA & 53 & 59 & M & 12 & AIDS \\
\hline 6 & 63 & M & 40 & 6.91 & Cardiac & 54 & 54 & M & 11 & AIDS \\
\hline 7 & 50 & M & 11 & 6.5 & Cardiac & 55 & 35 & M & 10 & AIDS \\
\hline 8 & 63 & $\mathrm{M}$ & 37 & 6.5 & Cardiac & 56 & 38 & $\mathrm{M}$ & 7 & AIDS \\
\hline 9 & 34 & $\mathrm{M}$ & 9 & 6.56 & MVA & 57 & 38 & M & 10 & AIDS \\
\hline 10 & 56 & $\mathrm{~F}$ & 29 & 6.78 & Cardiac & 58 & 39 & M & 12 & AIDS \\
\hline 11 & 56 & $\mathrm{~F}$ & 31 & 6.66 & Cardiac & 59 & 34 & M & 4 & AIDS \\
\hline 12 & 39 & $\mathrm{~F}$ & 24 & 6.88 & Cardiac & Mean & 44.7 & & 15.2 & \\
\hline
\end{tabular}

MDD

$\begin{array}{ll}13 & 32 \\ 14 & 47 \\ 15 & 56 \\ 16 & 33 \\ 17 & 45 \\ 18 & 24 \\ 19 & 56 \\ 20 & 44 \\ 21 & 34 \\ 22 & 53 \\ 23 & 45 \\ 24 & 45 \\ \text { Mean } & 42.8 \\ \text { MDD psychosis }\end{array}$

$25-48$

$26 \quad 40$

$27 \quad 28$

$28 \quad 28$

$29 \quad 62$

$30 \quad 28$

$31 \quad 32$

$32 \quad 63$

$33 \quad 51$

$34 \quad 40$

$35 \quad 35$

$36 \quad 36$

Mean $\quad 41.5$

HIV+

$38 \quad 45$

$39 \quad 57$

$40 \quad 35$

$41 \quad 55$

$42 \quad 57$

$43 \quad 54$

$44 \quad 49$

$\begin{array}{ll}45 & 37\end{array}$

$46 \quad 40$

$47 \quad 47$

$48 \quad 46$

Mean $\quad 46.6$
F 19

F 25

M 38

F 29

M 21

F 15

M 24

M 24

M 21

M 29

F $\quad 13$

23.6

6.8

6.88

6.59

6.86

6.9

6.61

6.59

6.52

6.79

6.64

6.75

6.58

6.71

F 24

F $\quad 40$

M 65

F $\quad 40$

F 19

M 31

F $\quad 36$

F 49

F

35.8

6.36

6.48

6.7

6.68

6.57

6.68

6.7

6.6

6.3

6.72

6.6

6.74

6.59

M 12

M 12

M 5

M 5

M 12

M 12

M 10

M 10

F 12

M 12

8.8
M 25

M 52

M 26

M 36

$\begin{array}{ll}\mathrm{F} & 32\end{array}$

F 1
Table 1 (continued)

$P M I$ postmortem interval, $M V A$ motor vehicle accident, $A A P$ acute alcohol poisoning, $O D$ drug overdose, $P E$ pulmonary embolism, $A I D S$ (complications due to) acquired immunodeficiency syndrome

FKBP5 genotyping of patient cohort

Genotyping of previously published alleles of the FKBP5 gene implicated in mood disorders was performed. Genomic DNA from the patients was isolated from the tissue following manufacturer's instructions of the DNeasy kit (Qiagen no. 69504). Genotyping was performed using $20 \mathrm{ng}$ DNA following manufacturer's protocols of the SNP Genotyping Assay from Applied Biosystems: an A/C substitution in the $3^{\prime}$ untranslated region of FKBP5 gene, SNP rs3800373, (Applied Biosystems Assay ID C_27489960_10), and a $\mathrm{C} / \mathrm{T}$ substitution in Intron 1 of the $F K B P 5$ gene, SNP rs1360780 (Applied Biosystems Assay ID C_8852038_10). A five by three table was constructed of the possible genotypes with the five test groups and Chi-square analysis compared the expected genotype frequencies with observed. Expected frequencies are determined by genotype frequency in North American Caucasian population deposited in NCBI SNP database; for rs3800373 record ID ss2334697 and for rs1360780, record ID ss4777328 was used (Haga et al. 2002). Two by five tables were constructed and allelic frequencies and Chi-square analysis compared the expected versus observed frequencies.

AIDS

AIDS

AIDS

AIDS

AIDS

AIDS

AIDS

AIDS

AIDS

AIDS

AIDS

\section{Results}

Immunophilin gene expression

In order to determine whether age, PMI, and brain $\mathrm{pH}$ were possible confounders, tests for correlation were performed. Since gene expression is calculated as Fold - Change $=2^{-\Delta \Delta \mathrm{CT}}$, a value of 0.5 corresponds to a 


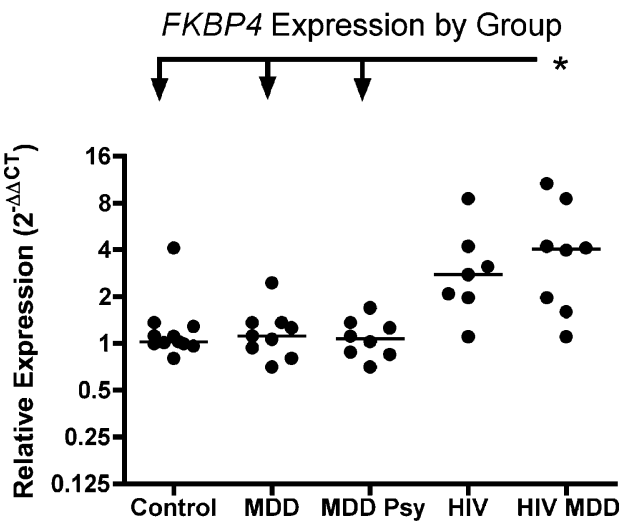

Fig. 1 FKBP4 and FKBP5 expression is increased in HIV and HIV with MDD. Gene expression in the cortical gray matter was determined by qPCR using the $\triangle \triangle \mathrm{CT}$ method and normalizing the gene of interest to GAPDH expression. Fold-Control is plotted for

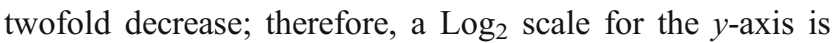
used so that 0.5 and 2 are equidistant from baseline; regression analyses for correlation account for this. Expression was plotted versus age, PMI, and brain $\mathrm{pH}$, and Pearson's test for correlation was performed. For all three characteristics, there were no correlations with gene expression, $R<0.50$

In examining Table 1 , one can see that the $\mathrm{HIV}^{+}$ specimen obtained from the CNTN had more males. The HIV-negative groups obtained from the Stanley Foundation had statistically indistinguishable male/female compositions. $t$ tests comparing expression in non-HIV males to expression in non-HIV females of the two immunophilin genes found no difference between the sexes with respect to immunophilin expression, showing $95 \%$ confidence intervals interquartile ranges. Since there was no difference between male and female expression of FKBP4 $(p=0.179)$ and FKBP5 ( $p=0.716)$, sex is unlikely to be a confounder to the analysis.

In order to represent the natural variation in the population at large, fold control was calculated against the median $\triangle \mathrm{CT}$ value from the control group. Therefore, half of the controls appear above and half below 1. As shown in Fig. 1, the majority of patients in the two $\mathrm{HIV}^{+}$populations showed higher expression of FKBP4 and FKBP5 above the control median. $\mathrm{HIV}^{+}$patients exhibited a fold increase of $3.4 \pm 2.3$ (mean $\pm 95 \%$ confidence interval), and $\mathrm{HIV}^{+} \mathrm{MDD}$ patients exhibited a fold increase of $4.5 \pm 2.9$ for the FKBP4 gene. $\mathrm{HIV}^{+}$patients showed 4.0 \pm 2.3 -fold increase over control, and $\mathrm{HIV}^{+} \mathrm{MDD}$ patients showed 5.6 \pm 3.5 -fold increase over control for the FKBP5 gene. In the $\mathrm{HIV}^{+} \mathrm{MDD}$ population, $F K B P 5$ was more variable, ranging from 0.7 - to 12.0 -fold change, while the $\mathrm{HIV}^{+}$ranged from 1.4- to 6.3-fold increase. In MDD, MDD/Psyc, HIV, and HIV/MDD, gene expression of both immunophilins is more variable. In the $\mathrm{HIV}^{-} \mathrm{MDD}$ group, the trend is toward

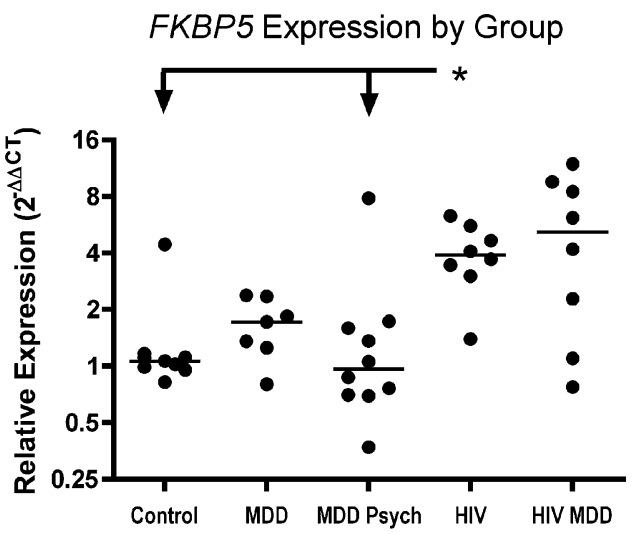

each of the study groups; circles represent individual patients, and bars represent median within the group. Kruskal-Wallace rank sums test with Dunn's multiple comparison tested for significant difference among the groups $(* p<0.05)$

increased expression of FKBP5 with median expression 1.7-fold higher than control; however, no change is apparent for FKBP4 in the HIV MDD population. For the FKBP4 gene, $\mathrm{HIV}^{+}$patients exhibited a fold increase of $3.2 \pm$ 2.6 (mean \pm standard deviation), and $\mathrm{HIV}^{+} \mathrm{MDD}$ patients exhibited a fold increase of $3.1 \pm 3.3$. For the FKBP 5 gene, $\mathrm{HIV}^{+}$patients showed $13.5 \pm 15.8$-fold increase over control, and $\mathrm{HIV}^{+} \mathrm{MDD}$ patients showed 9.6 \pm 12.7 -fold increase over control. In the $\mathrm{HIV}^{+} \mathrm{MDD}$ population, FKBP5 was more variable in the patient populations, ranging from 0.7- to 44.3-fold change in $\mathrm{HIV}^{+} \mathrm{MDD}$ and in $\mathrm{HIV}^{+}$from 1.4- to 50-fold increase. In the HIV-negative MDD group,

\section{Correlation FKBP4 v FKBP5}

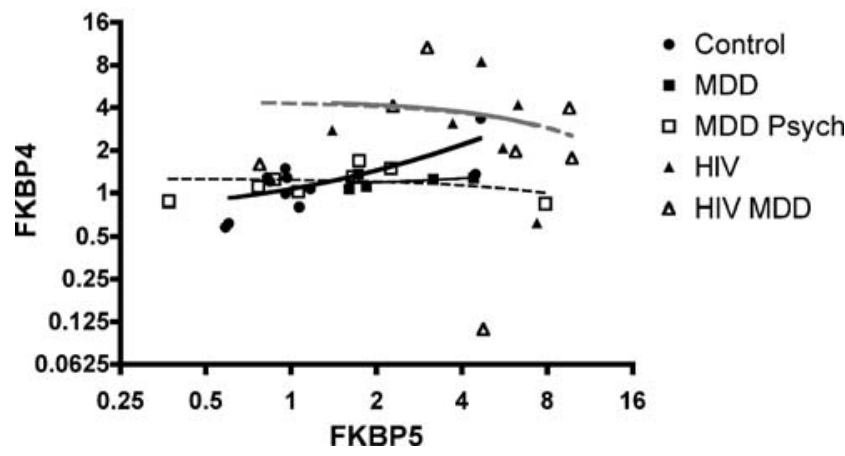

Fig. 2 Correlation of FKBP4 and FKBP5 expression in frontal cortex separated by group. Gene expression from the frontal cortex of patients listed in Table 1 is determined by qPCR using the $\Delta \Delta \mathrm{CT}$ method and normalizing the gene of interest to GAPDH. Fold-Control is plotted for each of the study group, plotting the FKBP4 expression on the $y$-axis and FKBP5 expression on the $x$-axis for each individual patient. For the control (circles, thick line) group, Pearson's test for correlation showed a correlation between FKBP4 and FKBP5 $(R=$ 0.745 ). For the MDD (filled squares, black thin line) and MDD/Psych (empty squares, black dashed line) groups, FKBP4 and FKBP5 were not correlated $(R=0.389$ and -0.287$)$. For both HIV (filled triangles, gray thin line) and HIV/MDD (empty triangles, gray dashed line), there was no correlation $(R=-0.178$ and -0.203 , respectively) 


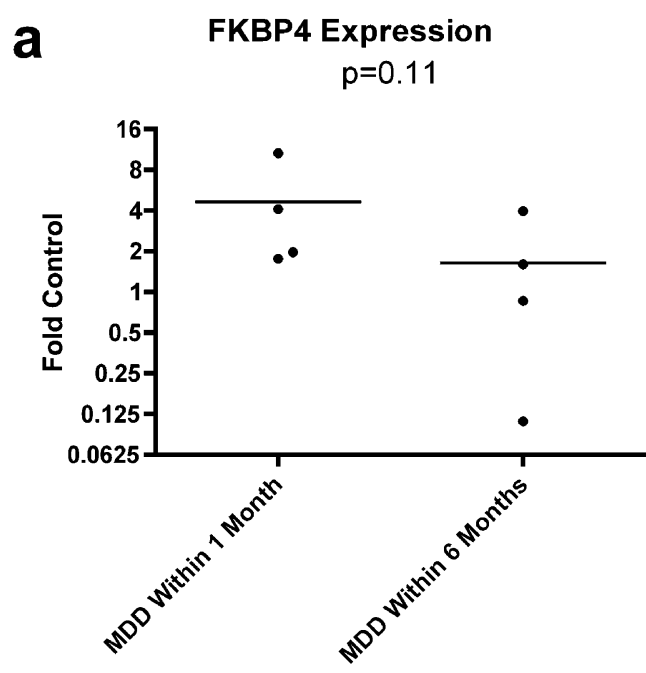

Fig. 3 Expression of $F K B P 4$ (a) and FKBP5 (b) in the CNTN groups separated by most recent episode. Gene expression from frontal cortex of patients for whom MDD clinical data were available is determined by $\mathrm{qPCR}$ using the $\triangle \triangle \mathrm{CT}$ method and normalizing the gene of interest to GAPDH expression. Patients are divided based on MDD

the trend is toward increased expression of FKBP5 with median expression $2.4-$-fold higher than control; however, no change is apparent for $F K B P 4$ in this group. In comparing $\mathrm{HIV}^{+}$to $\mathrm{HIV}^{-}$, both FKBP4 and FKBP5 genes are expressed at higher levels.

One subject was diagnosed with HAD, no. 49, and had the lowest expression of FKBP4 in the HIV/MDD group, 0.14-fold of control and an increase in FKBP5, 4.6-fold of control. Two subjects in the HIV group were diagnosed with MCMD; subjects no. 41 and no. 45. Patient no. 41 had the highest $F K B P 4$ expression in the HIV group, 8.5-fold for FKBP4 and 4.6-fold for FKBP5 over control. Patient no. 45 was 2.7 - and 1.4-fold over control for FKBP4 and $F K B P 5$, respectively. It is interesting that the patients with cognitive impairment were outliers in some respects; the only patient with HAD correlated with the lowest FKBP4 expressor in the HIV/MDD group, and one patient with

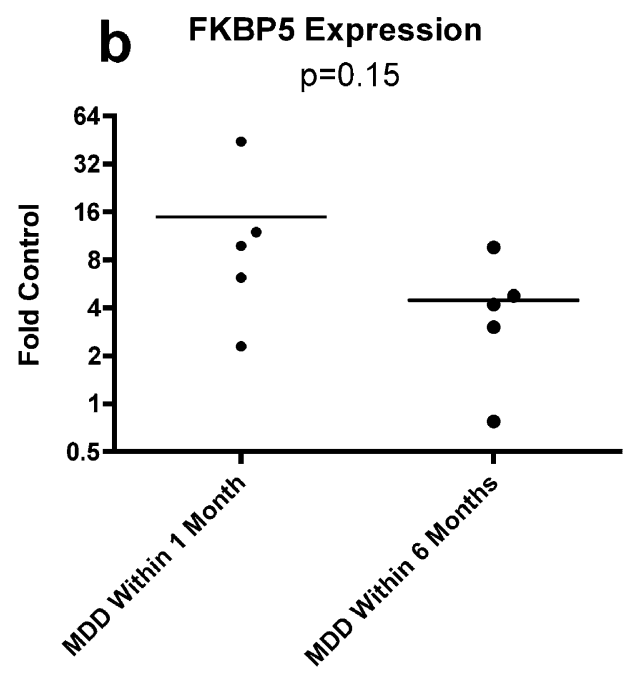

episode within 1 month or within 6 months and Fold-Control plotted; circles represent individuals, and bars represent median. Student's $t$ test comparing the two groups' expression showed a nonsignificant trend based on most recent MDD episode for expression of FKBP4 $p=0.11$ (a) or FKBP5 $p=0.15$ (b)

MCMD correlated to the highest FKBP4 expression in the HIV group. These three subjects were not outliers in their groups with respect to FKBP5 expression.

We also hypothesized that there would be a balance between FKBP4 and FKBP5 expression, and the balance would be disrupted in MDD, MDD/Psychosis, or HIV/ MDD. In Fig. 2, we plotted the FKBP4 expression on the $y$ axis and FKBP5 on the $x$-axis and analyzed the possible correlation in expression of the two genes, separating the groups. Pearson's test for correlation showed a correlation between FKBP4 and FKBP5 $(R=0.745)$ expression in the control group (circles), which is absent in all other groups. In the MDD (filled squares) and MDD/Psych (empty squares) groups, no correlation was observed between FKBP4 and FKBP5 ( $R=0.389$ and -0.287 , respectively). Even though expression was elevated in both HIV (filled triangles) and HIV/MDD (empty triangles), the correlation

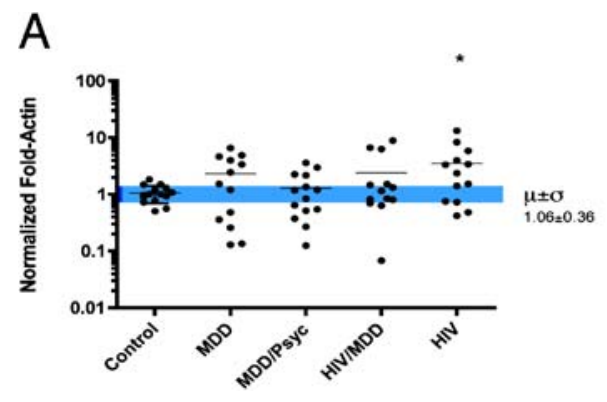

B
Fig. 4 FKBP52 protein levels are increased in the HIV group. Cases representing each study group were included in every gel. After transfer and immobilization on PVDF membrane, proteins were probed by Western blot for FKBP52; representative blot showed in b. Membranes were stripped of antibodies and reprobed for actin.
Values are normalized to controls on their respective gels, Normalized Fold-Actin $=$ Fold-Actin $n_{\text {Patient }} /$ Fold-Actin $_{\text {ControlMean }}$. The area within the bar delineates the standard deviation about the mean $(\mu \pm \sigma)$. Kruskal-Wallace and Dunn's multiple comparison test were used to test for significance $(* p<0.05 ; \mathbf{a})$ 


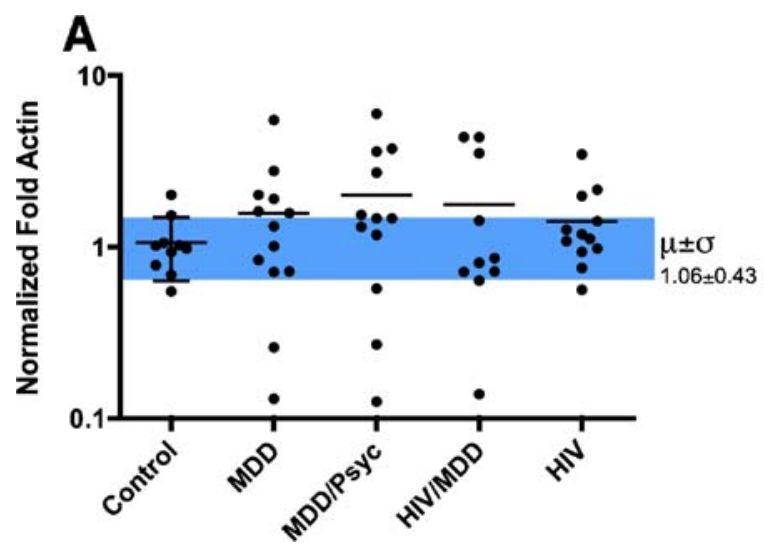

Fig. 5 FKBP51 protein levels are altered in MDD groups compared to control. Values were normalized to controls on their respective gels, Normalized Fold-Actin $=$ Fold-Actin Patient $_{\text {Fold-Actin }}$ ControlMean. The area within the bar delineates the standard deviation about the mean

of the two genes observed in the control group was no longer present when looking at the separate diagnostic groups $(R=-0.178$ and -0.203$)$.

In the tissue acquired from the CNTN, we had available patient history of the MDD and MDD/Psych patients regarding their most recent MDD ,episode and it was either within the last month or 6 months before death. The FKBP4 and FKBP5 expression was higher in the MDD patients who had MDD episodes within 1 month of death, but did not reach statistical significance, $p=0.11$ and $p=0.15$, respectively (Fig. 3).

\section{Protein analysis}

The protein analysis of FKBP52 and 51 illustrated in Figs. 4 and 5, respectively, showed a wide variation in expression of both immunophilins in the control group, indicating that expression varies in the population at large and likely is dependent on many factors. FKBP52 was elevated in HIV Group compared to control patients, $p=$ 0.0084. Compared to HIV without MDD, the HIV/MDD group, although more variable than the control, had lower levels of FKBP52. Amongst the MDD and MDD/Psychosis Groups, expression of FKBP52 is more variable than control, with the MDD mean above the standard deviation of the mean control. FKBP51 showed higher mean protein expression in the MDD, MDD/Psych, and HIV/MDD groups, however, not statistically significant; there were patients both above and below the standard deviation of the control group.

Single nucleotide polymorphism analysis

Using the allelic discrimination assay, we tested two polymorphisms: rs38000373, an $\mathrm{A}$ to $\mathrm{C}$ transversion
B

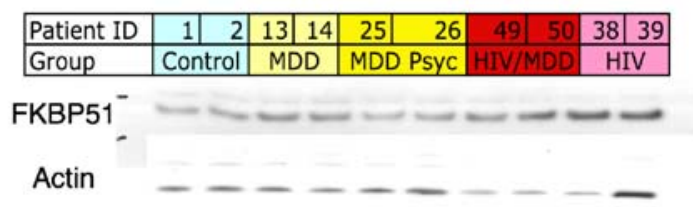

$(\mu \pm \sigma)$. Kruskal-Wallace and Dunn's multiple comparison test were used to test for significance at $p<0.05$ (a). Representative blot is showed in $\mathbf{b}$

substitution in the $3^{\prime}$ untranslated region ( $3^{\prime}$ UTR) of the FKBP5 gene and rs1360780, which is a $\mathrm{C}$ to $\mathrm{T}$ transition substitution in the second intron of the FKBP5 gene. The rs 1360780 polymorphism is located in a region where transcription factors may bind, where hormone response elements (HREs) are located as shown in Fig. 6, which may alter transcription and have been implicated in mood disorders (Hubler and Scammell 2004; Billing et al. 2007). The rs3800373 SNP is located in a region which may alter stability and half-life of the mRNA molecule.

The SNP rs3800373 had associations with MDD and MDD with Psychosis. The heterozygous genotype, AC, and the minor allele homozygous $\mathrm{CC}$ were significantly more frequent than expected based on Hardy-Weinberg assumptions of the North American Caucasian population and previously published allelic and genotype frequencies (Table 2; Haga et al. 2002). The HIV ${ }^{+}$MDD did not have the same deviation from expected as the HIV-negative MDD group. The MDD/Psych group had increased

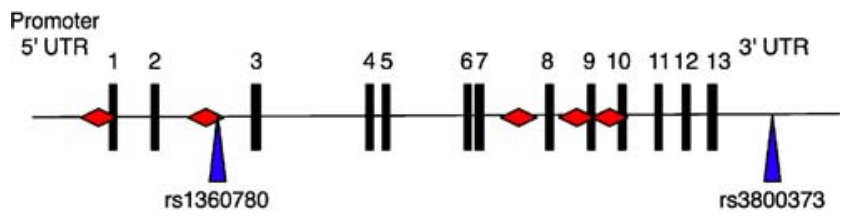

Hormone Response Elements

Fig. 6 Transcription factor binding sites and SNP locations on FKBP5 gene. The genomic organization of the FKBP5 gene is illustrated; exons are numbered with black bars; distance between bars represents relative lengths of the introns. The locations of the polymorphisms rs 1360780 and rs 3800373 are indicated by triangles, and the locations of hormone-responsive elements are indicated with diamonds (information from Hubler and Scammell 2004) 
Table 2 Genotype frequencies for SNPs rs3800373 and rs1360780 of FKBP5 gene

\begin{tabular}{|c|c|c|c|c|c|c|c|c|c|c|c|c|c|c|}
\hline & \multicolumn{7}{|c|}{ rs3800373 } & \multicolumn{7}{|c|}{ rs 1360780} \\
\hline & \multicolumn{3}{|c|}{ Expected } & \multicolumn{3}{|c|}{ Observed } & \multirow{2}{*}{$\begin{array}{l}\chi^{2} \\
p \text { value }\end{array}$} & \multicolumn{3}{|c|}{ Expected } & \multicolumn{3}{|c|}{ Observed } & \multirow{2}{*}{$\begin{array}{l}\chi^{2} \\
p \text { value }\end{array}$} \\
\hline & AA & $\mathrm{AC}$ & $\mathrm{CC}$ & $\mathrm{AA}$ & $\mathrm{AC}$ & $\mathrm{CC}$ & & AA & $\mathrm{AC}$ & $\mathrm{CC}$ & $\mathrm{AA}$ & $\mathrm{AC}$ & $\mathrm{CC}$ & \\
\hline Control $(n=13)$ & 5.4 & 7 & 0.5 & 8 & 4 & 1 & 0.232 & 5.4 & 7 & 0.5 & 8 & 4 & 1 & 0.232 \\
\hline $\operatorname{MDD}(n=11)$ & 5 & 6.5 & 0.5 & 7 & 2 & 2 & 0.015 & 5 & 6.5 & 0.5 & 7 & 2 & 2 & 0.015 \\
\hline MDD Psych $(n=10)$ & 4.6 & 6 & 0.5 & 2 & 6 & 2 & 0.037 & 4.6 & 6 & 0.5 & 2 & 6 & 2 & 0.037 \\
\hline $\operatorname{HIV}(n=9)$ & 3.8 & 4.9 & 0.4 & 2 & 6 & 1 & 0.35 & 3.8 & 4.9 & 0.4 & 2 & 6 & 1 & 0.35 \\
\hline HIV MDD $(n=8)$ & 3.3 & 4.3 & 0.3 & 3 & 5 & 0 & 0.79 & 3.3 & 4.3 & 0.3 & 3 & 5 & 0 & 0.79 \\
\hline
\end{tabular}

Genotypes were determined from genomic DNA by allelic discrimination assay described in "Materials and methods". Based on the published allelic frequencies for rs3800373: $f(\mathrm{~A})=0.688, f(\mathrm{C})=0.312$ and for rs1360780: $f(\mathrm{C})=0.758, f(\mathrm{~T})=0.242$, Expected genotype frequencies are calculated based on Hardy-Weinberg equilibrium and compared to Observed genotypes for the study groups. $\chi^{2}$ analysis comparing Expected with Observed values determines significant deviation in the patient groups from the population; significance is considered at $p<0.05$

frequency of the $\mathrm{C}$ allele compared with the North American Caucasian population and the control group in this study (Table 3 ).

In the, genotype frequencies of the Intron II polymorphism were significantly different in the MDD/Psych group from North American Caucasian population. The AC and $\mathrm{CC}$ genotypes were increased in the MDD/Psych as well as significant increase in the minor, $\mathrm{C}$, allele (Tables 2 and 3). A possible confounder to this analysis is that the control and the HIV populations differed from the Expected counts.

\section{Discussion}

The observation of unequal variance of transcriptional levels of both FKBP4 and FKBP5 among the groups may indicate a nonuniform distribution in the general population and suggests that multiple mechanisms can be involved. Nonetheless, the trends in variation in the MDD, MDD/Psychosis, HIV, and HIV/MDD groups suggest that the interplay between FKBP51 and FKBP52 in modulating the effects of cortisol at the cellular level may be of consequence.

Our observation of higher FKBP5 gene transcription levels in the MDD group that did not reach statistical significance (Fig. 1), combined with previous reports that FKBP5 is responsive to cortisol (Davies et al. 2002), likely indicates increased expression in response to hypercortisolemia in MDD. In HIV patients, immunophilin expression is clearly elevated, probably in response to chronic inflammation and microglial activation. Achim and Avramut showed higher expression of the immunophilin FKBP12 in the HIV-infected brain (Avramut and Achim 2003). If the immunophilins share similar promoter regions, containing SP1 binding elements and CCAAT boxes, it would make sense that immunophilins genes would be upregulated by overlapping mechanisms.

FKBP5 transcript was much more variable in $\mathrm{HIV}^{+} \mathrm{MDD}$ than $\mathrm{HIV}^{+}$, which could indicate two things. First, that transcription of FKBP5 would be elevated in direct response to hypercortisolemia, and second, that there is a

Table 3 Allelic frequencies for SNPs rs3800373 and rs1360780 of FKBP5 gene

\begin{tabular}{|c|c|c|c|c|c|c|c|c|c|c|}
\hline & \multicolumn{5}{|c|}{ rs3800373 } & \multicolumn{5}{|c|}{ rs 1360780} \\
\hline & \multicolumn{2}{|c|}{ Expected } & \multicolumn{2}{|c|}{ Observed } & \multirow{2}{*}{$\begin{array}{l}\chi^{2} \\
p \text { value }\end{array}$} & \multicolumn{2}{|c|}{ Expected } & \multicolumn{2}{|c|}{ Observed } & \multirow{2}{*}{$\begin{array}{l}\chi^{2} \\
p \text { value }\end{array}$} \\
\hline & $\mathrm{A}$ & $\mathrm{C}$ & $\mathrm{A}$ & $\mathrm{C}$ & & $\mathrm{C}$ & $\mathrm{T}$ & $\mathrm{C}$ & $\mathrm{T}$ & \\
\hline Control $(n=26)$ & 18 & 8 & 20 & 6 & 0.371 & 20 & 6 & 17 & 9 & 0.215 \\
\hline $\operatorname{MDD}(n=22)$ & 15 & 7 & 16 & 6 & 0.691 & 18 & 6 & 15 & 9 & 0.128 \\
\hline MDD Psych $(n=20)$ & 14 & 6 & 10 & 10 & 0.07 & 17 & 5 & 12 & 10 & 0.02 \\
\hline HIV $(n=18)$ & 12 & 6 & 10 & 8 & 0.225 & 14 & 4 & 9 & 9 & 0.011 \\
\hline HIV MDD $(n=16)$ & 11 & 5 & 11 & 5 & 0.997 & 12 & 4 & 11 & 5 & 0.51 \\
\hline
\end{tabular}

Genotypes were determined from genomic DNA by allelic discrimination assay described in "Materials and methods". Based on the published allelic frequencies for $\mathrm{rs} 3800373$ : $f(\mathrm{~A})=0.688, f(\mathrm{C})=0.312$ and for $\mathrm{rs} 1360780: f(\mathrm{C})=0.758, f(\mathrm{~T})=0.242$, Expected allele frequencies are calculated based on group size and compared to Observed genotypes for the study groups. $\chi^{2}$ analysis comparing Expected with Observed values determines significant deviation in the patient groups from the population; significance is considered at $p<0.05$ 
subgroup of patients whose neurons did not increase expression of FKBP5 concordantly to compensate resulting in abnormally high GR signaling rendering them susceptible to the effects of the glucocorticoid cascade.

The SNP, rs1360780, located in Intron II, which contains HREs, potential alternative promoter regions, and distal enhancer elements (Hubler and Scammell 2004), had genotype frequencies significantly different from expected in control, MDD/Psychosis, and HIV. After genotyping the FKBP5 gene for two SNPs, we found that rs 3800373 CC genotype is higher in the MDD and MDD/Psychosis group (Table 2); this SNP is located in the $3^{\prime}$ UTR of the mRNA and could potentially affect protein translation. In Fig. 5, we show higher protein levels of the FKBP5 gene product, FKBP51, in portions of the MDD and MDD/Psychosis groups as compared to control, while we did not find significant changes in transcription levels; it may be that this 3'UTR polymorphism affects translation efficiency. Our data analysis methods, based on comparing to controls, preclude determining whether higher protein amounts are found in the $\mathrm{C}$ allele, but it would be an interesting next step. Furthermore, new research on micro-RNAs show that mRNA stability, based on micro-RNA binding in 3'UTRs, affects the half-life of a particular transcript (Lai 2002); this would be another possibility of yet another level of modulation of cellular FKBP51 levels and thereby modulation of glucocorticoid signaling and HPA axis function.

It should be noted that our design study was correlative in analyzing gene and protein expressions in postmortem analyses; the results will be correlative in nature. We cannot determine whether FKBP51 and FKBP52 are dysregulated in the frontal cortex as a process resulting from MDD or as part of a process that led to MDD. Further, we do not know the causes of the increase observed to correlate to HIV infection; we hypothesize it to occur in neurons resulting from chronic inflammatory signals from infected monocytederived macrophages and microglia, from cytokine and chemokine secretion, since FKBP51 and FKBP52 are present in neurons of the frontal cortex, and neurons are not infected with HIV, but rather respond to signals from surrounding glia. However, it may be due to part of a cellular "stress response" from shedded viral particles.

We hypothesized that FKBP51, as modulator of the nuclear translocation of the GR, would be lower in MDD. Instead, elevated FKBP51 at both the transcript and protein levels correlates with MDD. However, FKBP52, the adapter protein that facilitates active GR trafficking, was variable in both directions, elevated, and decreased in MDD and MDD/Psych. In contrast to FKBP5, FKBP4 fold change was not significantly increased in HIV, but in $\mathrm{HIV}^{+} \mathrm{MDD}$. Perhaps immunophilins as a class of chaperone and adapter proteins are upregulated during chronic inflammation by a common mechanism, and if the agonist, in this case $F K B P 5$, is not significantly upregulated to compensate for the increased ability of GR to be functionally active, cortical neurons would be rendered more susceptible to the effects of glucocorticoids and the cascade leading to the subtle pathologies of depression.

Acknowledgments This study was supported by grants MH076681 to CLA, MH79881 to IPE, T32 EB001026 to ETT. California NeuroAIDS Tissue Network: U01 MH83506.

We thank the Stanley Foundation for donation of brain tissue for this study.

This work was performed in part with the support of the Genomics Core at the UCSD Center for AIDS Research (AI36214) and the San Diego Veterans Medical Research Foundation.

The HIV Neurobehavioral Research Center (HNRC) is supported by Center award MH 62512 from NIMH.

The San Diego HIV Neurobehavioral Research Center [HNRC] group is affiliated with the University of California, San Diego, the Naval Hospital, San Diego, and the Veterans Affairs San Diego Healthcare System and includes the following: Director-Igor Grant, M.D.; Co-Directors-J. Hampton Atkinson, M.D., Ronald J. Ellis, M. D., Ph.D., and J. Allen McCutchan, M.D.; Center Manager-Thomas D. Marcotte, Ph.D., Melanie Sherman; Naval Hospital San DiegoBraden R. Hale, M.D., M.P.H. (P.I.); Neuromedical ComponentRonald J. Ellis, M.D., Ph.D. (P.I.), J. Allen McCutchan, M.D., Scott Letendre, M.D., Edmund Capparelli, Pharm.D., Rachel Schrier, Ph.D., Jennifer Marquie-Beck, Terry Alexander, R.N., Janis Durelle; Neurobehavioral Component-Robert K. Heaton, Ph.D. (P.I.), Mariana Cherner, Ph.D., Steven Paul Woods, Psy.D., David J. Moore, Ph.D., Matthew Dawson; Neuroimaging Component-Terry Jernigan, Ph.D. (P.I.), Christine Fennema-Notestine, Ph.D., Sarah L. Archibald, M.A., John Hesselink, M.D., Jacopo Annese, Ph.D., Michael J. Taylor, Ph.D., Brian Schweinsburg, Ph.D.; Neurobiology ComponentEliezer Masliah, M.D. (P.I.), Ian Everall, FRCPsych., FRCPath., Ph.D., Cristian Achim, M.D., Ph.D.; Neurovirology Component-Douglas Richman, M.D., (P.I.), David M. Smith, M.D.; International ComponentJ. Allen McCutchan, M.D., (P.I.); Developmental Component-Ian Everall, FRCPsych., FRCPath., Ph.D. (P.I.), Stuart Lipton, M.D., Ph.D.; Clinical Trials Component-J. Allen McCutchan, M.D., J. Hampton Atkinson, M.D., Ronald J. Ellis, M.D., Ph.D., Scott Letendre, M.D.; Participant Accrual and Retention Unit-J. Hampton Atkinson, M.D. (P.I.), Rodney von Jaeger, M.P.H.; Data Management Unit-Anthony C. Gamst, Ph.D. (P.I.), Clint Cushman (Data Systems Manager), Daniel R. Masys, M.D. (Senior Consultant); Statistics Unit—Ian Abramson, Ph.D. (P.I.), Florin Vaida, Ph.D., Christopher Ake, Ph.D.

Open Access This article is distributed under the terms of the Creative Commons Attribution Noncommercial License which permits any noncommercial use, distribution, and reproduction in any medium, provided the original author(s) and source are credited.

\section{References}

Avramut M, Achim CL (2003) Immunophilins in nervous system degeneration and regeneration. Curr Top Med Chem 3(12):13761382

Billing AM, Fack F, Renaut J, Olinger CM, Schote AB, Turner JD, Muller CP (2007) Proteomic analysis of the cortisol-mediated stress response in Thp-1 monocytes using dige technology. J Mass Spectrom 42(11):1433-1444

Binder EB, Salyakina D, Lichtner P, Wochnik GM, Ising M, Putz B, Papiol S, Seaman S, Lucae S, Kohli MA, Nickel T, Kunzel HE, 
Fuchs B, Majer M, Pfennig A, Kern N, Brunner J, Modell S, Baghai T, Deiml T, Zill P, Bondy B, Rupprecht R, Messer T, Kohnlein O, Dabitz H, Bruckl T, Muller N, Pfister H, Lieb R, Mueller JC, Lohmussaar E, Strom TM, Bettecken T, Meitinger T, Uhr M, Rein T, Holsboer F, Muller-Myhsok B (2004) Polymorphisms in Fkbp5 are associated with increased recurrence of depressive episodes and rapid response to antidepressant treatment. Nat Genet 36(12):1319-1325

Binder EB, Bradley RG, Liu W, Epstein MP, Deveau TC, Mercer KB, Tang Y, Gillespie CF, Heim CM, Nemeroff CB, Schwartz AC, Cubells JF, Ressler KJ (2008) Association of Fkbp5 polymorphisms and childhood abuse with risk of posttraumatic stress disorder symptoms in adults. JAMA 299(11):1291-1305

Bing EG, Burnam MA, Longshore D, Fleishman JA, Sherbourne CD, London AS, Turner BJ, Eggan F, Beckman R, Vitiello B, Morton SC, Orlando M, Bozzette SA, Ortiz-Barron L, Shapiro M (2001) Psychiatric disorders and drug use among human immunodeficiency virus-infected adults in the United States. Arch Gen Psychiatry 58(8):721-728

Christeff N, Gherbi N, Mammes O, Dalle MT, Gharakhanian S, Lortholary O, Melchior JC, Nunez EA (1997) Serum cortisol and dhea concentrations during Hiv infection. Psychoneuroendocrinology 22(Suppl 1):S11-S18

Cotter D, Landau S, Beasley C, Stevenson R, Chana G, MacMillan L, Everall I (2002a) The density and spatial distribution of gabaergic neurons, labelled using calcium binding proteins, in the anterior cingulate cortex in major depressive disorder, bipolar disorder, and schizophrenia. Biol Psychiatry 51(5):377-386

Cotter D, Mackay D, Chana G, Beasley C, Landau S, Everall IP (2002b) Reduced neuronal size and glial cell density in Area 9 of the dorsolateral prefrontal cortex in subjects with major depressive disorder. Cereb Cortex 12(4):386-394

Davies TH, Ning YM, Sanchez ER (2002) A new first step in activation of steroid receptors: hormone-induced switching of Fkbp51 and Fkbp52 immunophilins. J Biol Chem 277(7):4597-4600

Denny WB, Valentine DL, Reynolds PD, Smith DF, Scammell JG (2000) Squirrel monkey immunophilin Fkbp51 Is a potent inhibitor of glucocorticoid receptor binding. Endocrinology 141 (11):4107-4113

Garcia A, Steiner B, Kronenberg G, Bick-Sander A, Kempermann G (2004) Age-dependent expression of glucocorticoid- and mineralocorticoid receptors on neural precursor cell populations in the adult murine hippocampus. Aging Cell 3(6):363-371

Gawlik M, Moller-Ehrlich K, Mende M, Jovnerovski M, Jung S, Jabs B, Knapp M, Stoeber G (2006) Is Fkbp5 a genetic marker of affective psychosis? A case control study and analysis of disease related traits. BMC Psychiatry 6:52

Haga H, Yamada R, Ohnishi Y, Nakamura Y, Tanaka T (2002) Gene-based Snp discovery as part of the Japanese millennium genome project: identification of 190,562 genetic variations in the human genome. Single-nucleotide polymorphism. J Hum Genet 47(11):605-610

Hubler TR, Scammell JG (2004) Intronic hormone response elements mediate regulation of Fkbp5 by progestins and glucocorticoids. Cell Stress Chaperones 9(3):243-252

Ising M, Depping A-M, Siebertz A, Lucae S, Unschuld PG, Kloiber S, Horstmann S, Uhr M, Müller-Myhsok B, Holsboer F (2008) Polymorphisms in the Fkbp5 gene region modulate recovery from psychosocial stress in healthy controls. Eur J Neurosci 28 (2):389-398

Koenen KC, Saxe G, Purcell S, Smoller JW, Bartholomew D, Miller A, Hall E, Kaplow J, Bosquet M, Moulton S, Baldwin C (2005) Polymorphisms in Fkbp5 are associated with peritraumatic dissociation in medically injured children. Mol Psychiatry 10 (12):1058-1059

Lai EC (2002) Micro Rnas are complementary to 3' Utr sequence motifs that mediate negative post-transcriptional regulation. Nat Genet 30(4):363-364

Lekman M, Laje G, Charney D, Rush AJ, Wilson AF, Sorant AJM, Lipsky R, Wisniewski SR, Manji H, McMahon FJ, Paddock S (2008) The Fkbp5-gene in depression and treatment responsean association study in the sequenced treatment alternatives to relieve depression (Star*D) cohort. Biol Psychiatry 63(12):11031110

Porche DJ, Willis DG (2006) Depression in Hiv-infected men. Issues Ment Health Nurs 27(4):391-401

Reiter GS (2000) Comprehensive clinical care: managing hiv as a chronic illness. AIDS Clin Care 12(2):13-19

Sapolsky RM, Plotsky PM (1990) Hypercortisolism and its possible neural bases. Biol Psychiatry 27(9):937-952

Sinars CR, Cheung-Flynn J, Rimerman RA, Scammell JG, Smith DF, Clardy J (2003) Structure of the large Fk506-binding protein Fkbp51, an Hsp90-binding protein and a component of steroid receptor complexes. Proc Natl Acad Sci USA 100(3):868-873

Steiner JP, Dawson TM, Fotuhi M, Glatt CE, Snowman AM, Cohen N, Snyder SH (1992) High brain densities of the immunophilin Fkbp colocalized with calcineurin. Nature 358(6387):584-587

Valente SM (2003) Depression and Hiv disease. J Assoc Nurses AIDS Care 14(2):41-51

Westberry JM, Sadosky PW, Hubler TR, Gross KL, Scammell JG (2006) Glucocorticoid resistance in squirrel monkeys results from a combination of a transcriptionally incompetent glucocorticoid receptor and overexpression of the glucocorticoid receptor cochaperone Fkbp51. J Steroid Biochem Mol Biol 100(1-3):34-41

Wochnik GM, Ruegg J, Abel GA, Schmidt U, Holsboer F, Rein T (2005) Fk506-binding proteins 51 and 52 differentially regulate dynein interaction and nuclear translocation of the glucocorticoid receptor in mammalian cells. J Biol Chem 280(6):46094616 